\title{
Ferramentas de Projeto para análise da qualidade urbana: Relacionando forma, usos, densidade e configuração espacial na cidade de João Pessoa, Brasil.
}

\author{
Design tools to assess urban quality: Relating form, uses, density and spatial configuration in \\ João Pessoa city, Brazil.
}

\author{
Geovany Jessé Alexandre da Silva \\ Universidade Federal da Paraíba, Brasil \\ galexarq.ufpb@gmail.com \\ Carlos Alejandro Nome \\ Universidade Federal da Paraíba, Brasil \\ carlosnome@gmail.com
}

\author{
Lucy Donegan \\ Universidade Federal da Paraíba, Brasil \\ lucydonegan@gmail.com
}

\begin{abstract}
This paper describes an experience in a Graduate course Architecture and Urbanism that used computational tools to analyze urban quality - considering form, uses, density and spatial configuration (based on visual and fields) - in different urban areas in the city of João Pessoa. Understanding that the city is a problem in organized complexity, different aspects condition the quality of use of spaces and reveal urban dynamics. Urban analysis aided by computational tools revealed successful in characterizing different problems and potentialities that can lay the foundation for interventions with more urban quality.
\end{abstract}

Keywords: Design computational tools; Study of urban form, uses and density; Urban space performance; Spatial configuration.

\section{Introdução}

Este artigo relata o processo experimental do uso de métodos e ferramentas aplicadas à análise da qualidade urbana, considerando especialmente os aspectos da forma, usos, densidade e configuração espacial, focando em campos visuais e de movimento. A cidade é um problema em complexidade organizada (Jacobs, 1992), e a relação espacial é intrínseca à configuração da qualidade e à dinâmica da vida urbana. Para compreender essa dinâmica complexa do espaço construído e as inter-relações de uso e apropriação dos lugares, em face dos diversos âmbitos de análise possíveis, demandam procedimentos metodológicos quantiqualitativos. Tais procedimentos poderiam ser capazes de potencializar a interpretação da cidade e frações urbanas, transformando-se em diretrizes ou programa de projeto.

No campo da Arquitetura e Urbanismo contemporâneo, as ferramentas computacionais aplicadas permitem subsidiar decisões projetuais em diversas escalas, do urbano ao edificado.

Como estudo-piloto, foi utilizada a disciplina do mestrado da Pós-Graduação em Arquitetura e Urbanismo intitulada "Qualidade e Avaliação do Ambiente Construído na Escala Urbana", da Universidade Federal da Paraíba, em João Pessoa-PB, Brasil. O objeto de análise se deu em bairros da capital paraibana, escolhido conforme a moradia e vivência de cada estudante. Para tanto, recorreu-se a análise dos bairros a partir dos critérios formais e espaciais, e ao final da disciplina, realizou-se uma oficina experimental de desenho urbano em uma área comum para todos os estudantes tomando por base as análises prévias.

\section{Método e procedimentos}

Dividiu-se o método analítico quanti-qualitativo (portanto, misto) em dois módulos após uma introdução mais teórica. 0 Primeiro módulo teórico/aplicado: Análise sistêmica e instrumentos de avaliação baseou-se no Estudo da Forma, Densidade e Usos Urbanos. Para análise da Forma Urbana, utilizou-se o procedimento de decomposição formal (sistêmica e elementar), a partir da teoria de Coelho (2014), e cálculo/estudo de índices urbanísticos (Índice de Aproveitamento, Taxa de Ocupação, Índice de espaços abertos, Gabaritos, Usos, Densidades edificadas e populacionais). Para se compreender a dinâmica de usos, utilizou-se indicadores de uso misto de Van de Hoek (2008) e análise espacial por meio do Spacemate, de Berghauser Pont \& Haupt (2009). Nesta etapa cada aluno selecionou um bairro da cidade para estudo.

O Segundo Módulo teórico/aplicado-instrumental foi a Análise sistêmica do espaço - por instrumentos de avaliação da configuração espacial de campos visuais e de movimento potencial dos espaços abertos, analisando hierarquias de acessibilidade topológica urbana (Hillier, 1996; Hillier \& Hanson, 1984; Karimi, 2012), Nesta etapa, cada aluno selecionou um espaço público foco (praça ou rua) e estudou a configuração espacial do entorno caminhável (400m) e 
campos visuais do espaço público em foco. Medidas de acessibilidade topológica são visualizados em mapas uma escala cromática, de vermelho para as entidades mais rasas no sistema (ou mais integradas) até azul para as entidades mais profundas no sistema (ou mais segregados). Por fim, seguiu-se com um espaço de oficina para o desenho urbano propositivo em uma área comum para todos os alunos, visando a aplicação dos conceitos e análises do projeto (Figura 1).

As ferramentas digitais usadas foram: SIG (programa QuantumGIS'1), AutoCAD, Depthmap X², Rhino/Grasshopper.
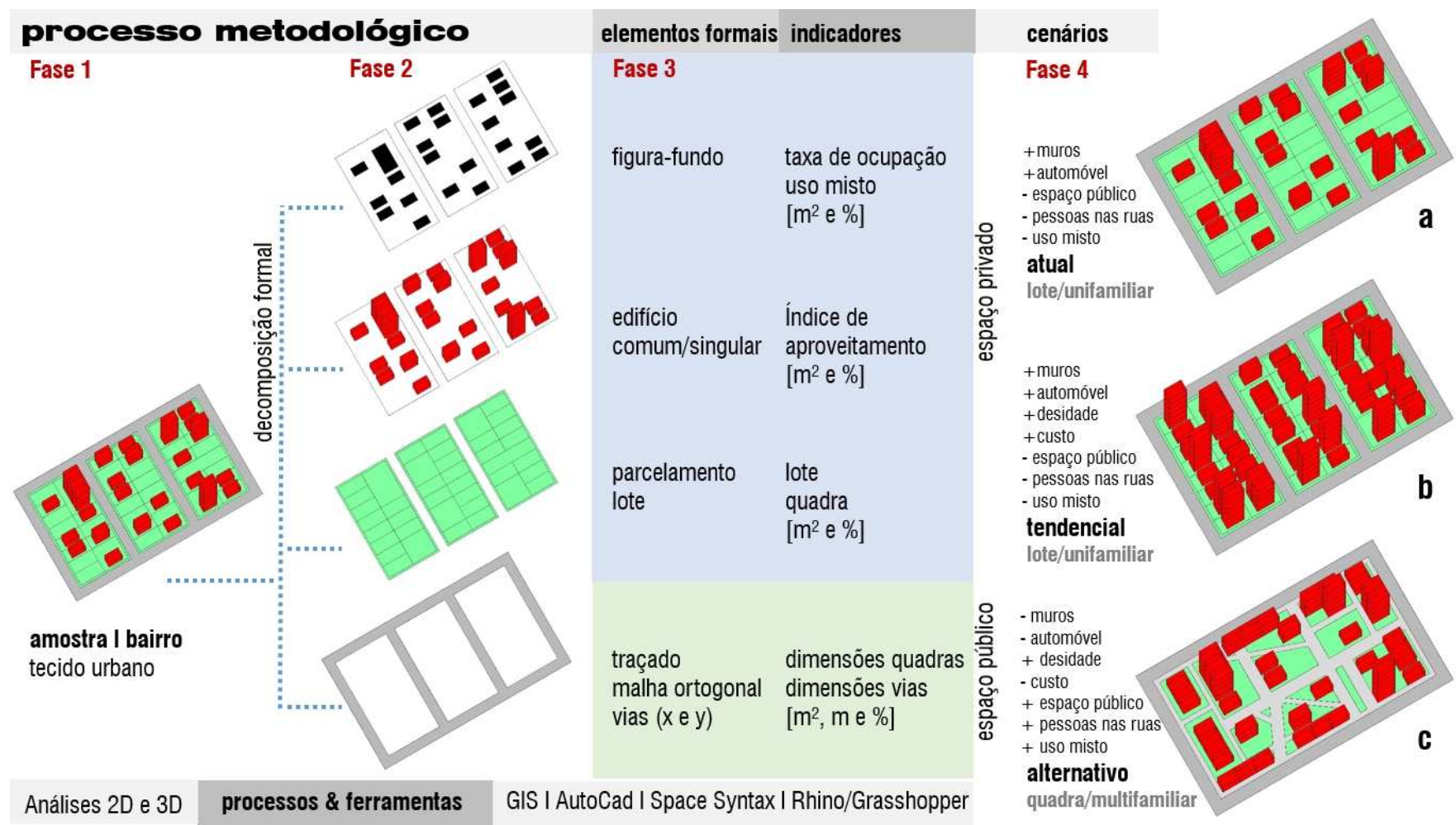

Figura 1 Síntese da Pesquisa: Procedimentos metodológicos e as fases de análise e cenários para as amostras urbanas simuladas em 2D e 3D. Fonte: Autores (2017).

\section{Revisão teórica}

A componente formal é um dos principais critérios passíveis de mensuração e caracterização por planejadores urbanos e regionais, e por arquitetos-urbanistas. Na geografia, na biologia, na engenharia ambiental e urbana, na arquitetura e no urbanismo, entre outros campos, a ocupação do espaço pode ser analisada sob diversos focos e ferramentas interpretativas. No caso do desenho urbano (ou projeto urbano), especificamente, o enlace qualitativo entre os espaços públicos e privados, assim como a relação da forma edificada e os vazios, são imprescindíveis para a potencialização da qualidade de vida. Contudo, a relação formal é, em geral, negligenciada pela gestão e política urbana. E assim se constituem as tais políticas urbanas e suas leis, que resultam em ocupações, projetos de bairros e edifícios que geralmente desconsideram o impacto das construções sobre a vida urbana e as pessoas que caminham na calçada (ou se escondem atrás dos muros das ruas), ignoram o impacto sobre os recursos naturais que envolvem

1 Programa de fonte aberta disponível em: www.qgis.org/ os loteamentos (ou são invadidos por estes), ou mesmo o grau de dispersão e densidade espacial dos conjuntos edilícios.

Essa lacuna de abordagem sobre o desenho urbano no planejamento da cidade deflagra problemas que poderiam ser evitados sob outras abordagens. A verticalização descontrolada (das áreas mais valorizadas) causa impactos no clima, segrega as famílias em condomínios fechados para a rua ou para a vida dos espaços públicos. A dispersão horizontal (em geral, de áreas periféricas urbanas) de baixa densidade também apresenta problemas impactando áreas verdes, demandando elevados custos de implementação e manutenção de infraestruturas. Esta dispersão também dificulta a acessibilidade aos equipamentos públicos, e agrava a mobilidade urbana como um todo. Entretanto estes dois modelos, o vertical e o disperso, são os mais realizados nas cidades brasileiras nas últimas décadas.

2 De fonte aberta desenvolvido por Turner, atualizado por Varoudis. 
A densidade urbana, em especial a densidade populacional, é um critério relevante que, comparado a outros processos de análise, pode agregar observações relevantes sobre a forma de ocupação e apropriação dos espaços da cidade (Bertaud \& Maupezzi 2003, Carmona et al 2007, Carmona \& Tiesdell 2003, Duany \& Plater-Zyberk 2001, Farr 2013, Frumkin 2002, Glaeser \& Kahn 2004, Jacobs 1992, Karssenberg et al 2015, Alexander 2013, Newmn \& Kenworthy 1989 e 1999). É necessário delimitar suas escalas de análise, e o objeto específico de mensuração, para evitar equívocos na delimitação de áreas mensuradas (como p.ex., a densidade líquida ou bruta) que podem interferir no cálculo final dos dados com variações importantes.

Entende-se que as decisões no campo do planejamento urbano e nas estratégias de enfrentamento ao rápido processo de urbanização são pontos chave na promoção da equidade social (Sennet, 2008), nos padrões de mobilidade (Bill Hillier, 2009), competitividade global e eficiência energética. Ainda há muitos estudos (Kenworthy \& Laube, 1999; Newman \& Kenworthy, 1989 e 1999; Frumkin, 2002; Camagni, Gibelli \& Rigamonti, 2002; Jackson, 2003; Glaser \& Kahn, 2004; Frank, 2006; Bart, 2010) que correlacionam o aumento do grau de emissão de gases estufas em paralelo à dispersão urbana de baixa densidade.

Há diversas incoerências quanto à forma de ocupação urbana recente. A verticalização acentuada, além de impactos climáticos e de infraestrutura, tem uma correlação direta com a incidência de doenças mentais (Alexander, 2013). Por outro lado, os conjuntos urbanos com edifícios estreitos, porém em blocos de até quatro pisos, de uso misto, com mais acessos à rua (embasamento ou "plints" com mais aberturas e acessíveis à rua) em quadras menores são melhores enquanto ao desenho urbano e densidade pois intensificam a relação das pessoas com a rua e entre si, como afirma Jane Jacobs (1992), Jan Gehl (2014), Christopher Alexander (2013) e Hans Karssenberg (2015). A dispersão urbana em baixa densidade, no caso dos condomínios de alta e média renda, é propagandeada como provendo, para o citadino, o convívio com o verde e maior "paz e tranquilidade"; nos casos dos conjuntos de baixa renda refletem uma atuação do mercado sobre o baixo custo da terra urbanizada sobre áreas periféricas (Villaça, 2001).

Entretanto, segundo Li \& Kanazawa (2016), a vida no campo em países mais desenvolvidos é mais feliz que na cidade, e a alta densidade pode diminuir o grau de felicidade das pessoas. Essa afirmação é corroborada por Berry \& Okulicz-Kozaryn (2009) e Easterlin, Angelescu, \& Zweig (2011). Mesmo na China, onde o rural é mais desigual economicamente em relação à cidade, a sensação subjetiva de bem-estar é maior no campo (Knight \& Gunatilaka, 2010). Mas, a sociedade contemporânea é a mais urbana da história. Assim, prover espaços que indiquem maior qualidade e otimizem recursos, incluindo espaciais, é uma das premissas mais relevantes para o desenho urbano atual.

De modo complementar, entendendo que a configuração espacial cria um campo provável de movimento, encontros e de campos visuais (B. Hillier, Burdett, Peponis, \& Penn, 1987), seu estudo pode destacar problemas e ensaiar possibilidades de intervenção. Notou-se, por exemplo, que determinados usos de maior porte e comerciais tendem a se situar em locais mais integrados do sistema, tomando proveito do seu movimento potencial (Hillier, 1996). Estudos mostraram evidências espaciais antes e no decorrer de projeto, permitindo ensaios mais aprofundados que resultaram em projetos mais bem-sucedidos no tempo em diversas escalas de intervenção urbana (Karimi, 2012).

A literatura sobre aspectos analíticos para entendimento do espaço urbano construído, é ampla e bem desenvolvida. No entanto ainda encontram-se lacunas nas questões referentes à definição do papel que estes métodos e ferramentas potencialmente têm no processo projetual urbano. É oportuno explorar a programação urbanística por critérios de desempenho urbano apoiados em parâmetros analíticos como os aqui descritos.

\section{Objeto de estudo e processos de análise}

Em princípio, definiram-se amostras de bairros de João Pessoa-PB, para possibilitar uma análise dos processos urbanos contemporâneos em diferentes aspectos, considerando as particularidades das escolhas de bairro por cada aluno (Tabela 1), a partir do critério de pertencimento do estudante à vizinhança selecionada. Foram definidos 3 módulos de trabalho a serem elencados a seguir.

Tabela 1: Áreas de estudo por mestrando e produtos de cada análise.

\begin{tabular}{|l|l|l|}
\hline Área de Estudo & Mestrando & Produtos \\
\hline Bairro Manaíra & Cássio V. C. de Sousa & Análises Formais, Usos, \\
Densidade Urbana. \\
Bairro Aeroclube & Cyro Visgueiro Maciel & Estudos de Configuração \\
\hline Jardim Oceania & Guilhardo B. M. de Carvalho & Espacial; \\
\hline Bairro Valentina & Lílian Leite Félix & $\begin{array}{l}\text { Uso de Ferramentas } \\
\text { Computacionais. }\end{array}$ \\
\hline Bairro Tambaú & Mayara Cynthia B. de Sousa \\
\hline
\end{tabular}

\section{Módulo 1}

No primeiro módulo [teórico/aplicado] se elaborou a análise sistêmica e se aplicou os instrumentos de avaliação da qualidade espacial e de usos, procedeu-se o Estudo da Forma, Densidade e, por fim, os Usos Urbanos das amostras de bairro selecionadas, como está apresentado o exemplo da Figura 2 (análise do bairro Aeroclube em João Pessoa-PB).

Quanto ao estudo da Forma Urbana, utilizou-se o procedimento de decomposição formal (sistêmica e elementar), adotada por Coelho (2014). Posteriormente, realizaram-se cálculos e estudos de índices urbanísticos, tais como Índice de Aproveitamento, Taxa de Ocupação, Índice de Espaços Abertos, Gabaritos, e, a partir destes dados pôde-se juntar as amostras em uma matriz espacial, conforme a teoria aplicada por Berghauser Pont \& Haupt (2009), no Spacemate/Spacematrix. 


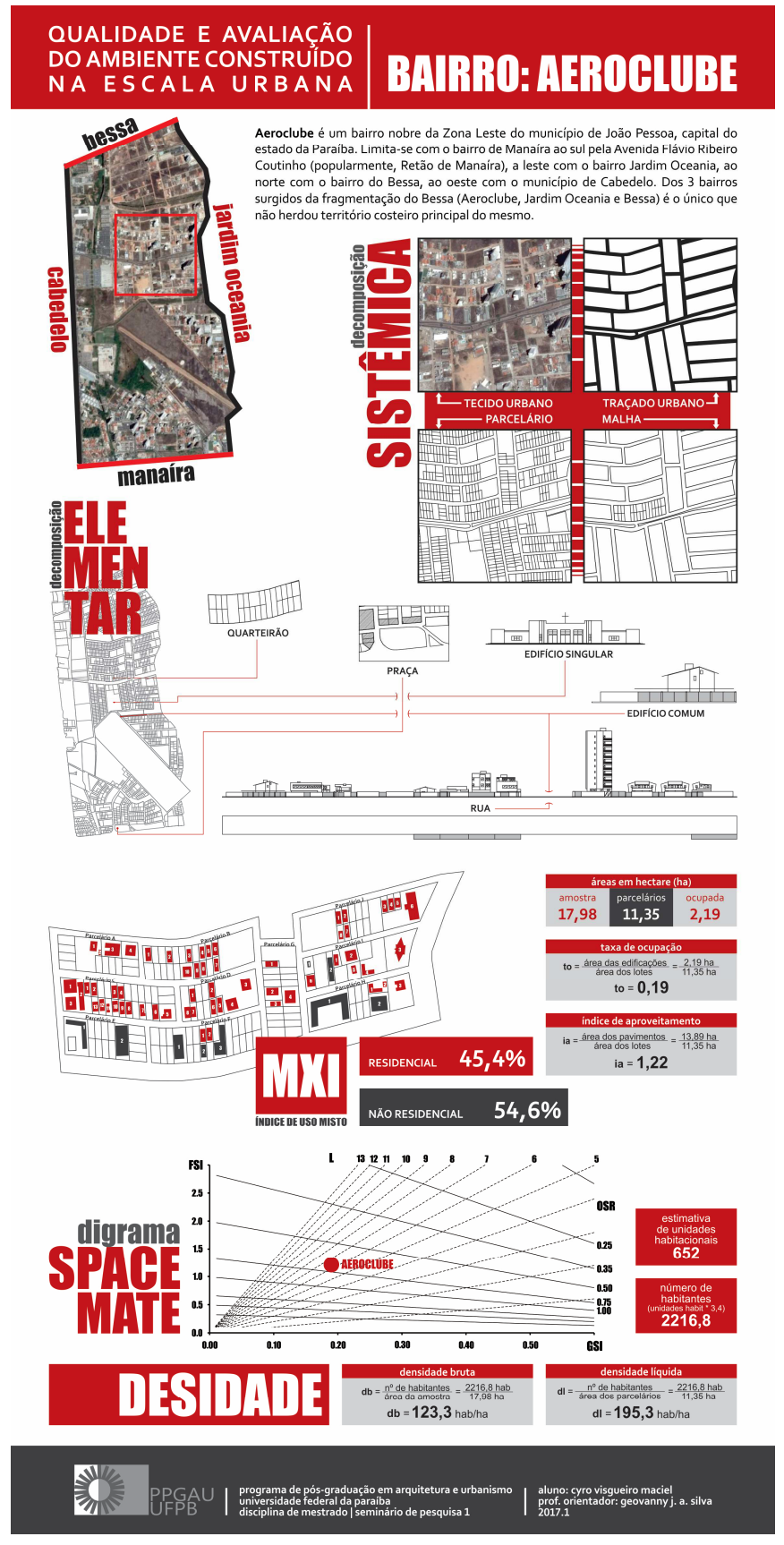

Figura 2 Painel final apresentado no Módulo 1 da disciplina, bairro Aeroclube (João Pessoa) no qual estão expostos a decomposição da forma urbana, o índice de uso misto (MXI), o diagrama da matriz espacial (Spacemate) e densidade urbana da amostra. Fonte: Cyro Visgueiro (2017).

A Densidade Urbana Bruta - a relação de habitantes por hectare total da amostra - e Líquida - relação de habitantes por áreas habitadas das quadras excluindo as vias e espaços públicos - foram os dados também calculados que possibilitaram mensurar o grau de presença de pessoas por bairro, que poderiam refletir em especificidades de uso dos espaços urbanos em cada situação.
Por fim, para compreender com maior precisão a dinâmica de usos e o potencial de urbanidade e vitalidade urbana das áreas estudadas, como exemplo metodológico de análise os indicadores de uso misto de Van de Hoek (2008), por meio do qual se pôde mensurar, a partir da área construída térrea das edificações, o percentual de usos residencial e não residencial por amostra de bairro. Em geral, neste quesito, o percentual que se aproxima de $50 \%$ (residencial/não residencial) é o mais desejável, pois aproxima-se do grau de urbanidade dos centros urbanos mais vivos e de maior dinâmica para a caminhabilidade.

Assim, aplicou-se a teoria do urbanismo e projeto por meio de ferramentas quantificáveis para os espaços da cidade.

\section{Módulo 2}

No segundo módulo [teórico/aplicado] os alunos selecionaram um espaço público do recorte estudado para desenvolver análises espaciais. Quase todos selecionaram uma praça (apenas um focou o uso em uma rua, já que no seu recorte não havia uma praça em uso). Os alunos estudaram relações de movimentos prováveis da configuração espacial em dois níveis: (a) veicular no entorno caminhável do espaço estudado, para interpretar algumas hierarquias das ruas no entorno do espaço público (b) pedonal no espaço público em foco, para entender movimento em uma escala mais local, e relacioná-lo aos campos visuais. Os campos visuais focaram o espaço público e ruas imediatas. Uma variação foi feita para imaginar possíveis cenários visuais ensaiando situações sem os muros existentes, deixando apenas as edificações, com vias de estimular ideias de intervenções. Tipos de interfaces público/privado também foram estudados, embora esta etapa não foi desenvolvida por todos (limitações de processamento e tempo).

Assim as seguintes análises foram desenvolvidas (i) Mapas axiais, analisando eixos de movimento potencial: (a) No entorno de $400 \mathrm{~m}$ para movimento veicula; (b) No espaço público e arredores (outro lado da rua) para movimento de pedestres; (ii) Mapas de campos visuais: (a) no espaço público com muros; (b) no espaço público mantendo apenas as edificações; (iii) Interfaces público-privado dos lotes frente ao espaço público em foco.

Ao final do módulo dois, pediu-se que os alunos relacionassem as análises dos dois módulos.

\section{Módulo 3}

Ao final da disciplina aconteceu uma oficina onde os alunos se juntaram para propor um desenho urbano em uma das áreas de estudo, considerando também parâmetros urbanísticos (legislação) da área, e considerando as análises descritas. Apontamentos iniciais de projeto apontaram cenários alternativos à ocupação tradicionalmente consolidada nas últimas décadas.

A seleção de uma área comum para todos os participantes foi feita a partir da discussão das amostras e dos bairros analisados. Decidiu-se por uma área pública do Bairro Oceania. A Oficina de Projeto estudou possíveis intervenções 
e cenários futuros, com a participação de alunos e professores da disciplina. Como suporte à oficina foram analisadas imagens de satélite, mapas, plantas da área, legislação urbana, e as simulações computacionais em 2D e 3D (Figura 3).

As discussões propositivas foram amparadas na definição qualitativa de critérios de desempenho urbano desejáveis nos termos das simulações prévias. Como: maior conectividade, e integração. Os resultados alcançados permitiram a compreensão de possíveis repercussões das intervenções propostas nos termos dos módulos 1 e 2 . Em decorrência destas discussões compreendeu-se também a necessidade da definição prévia de critérios de desempenho urbano mensuráveis para avaliação dos resultados.
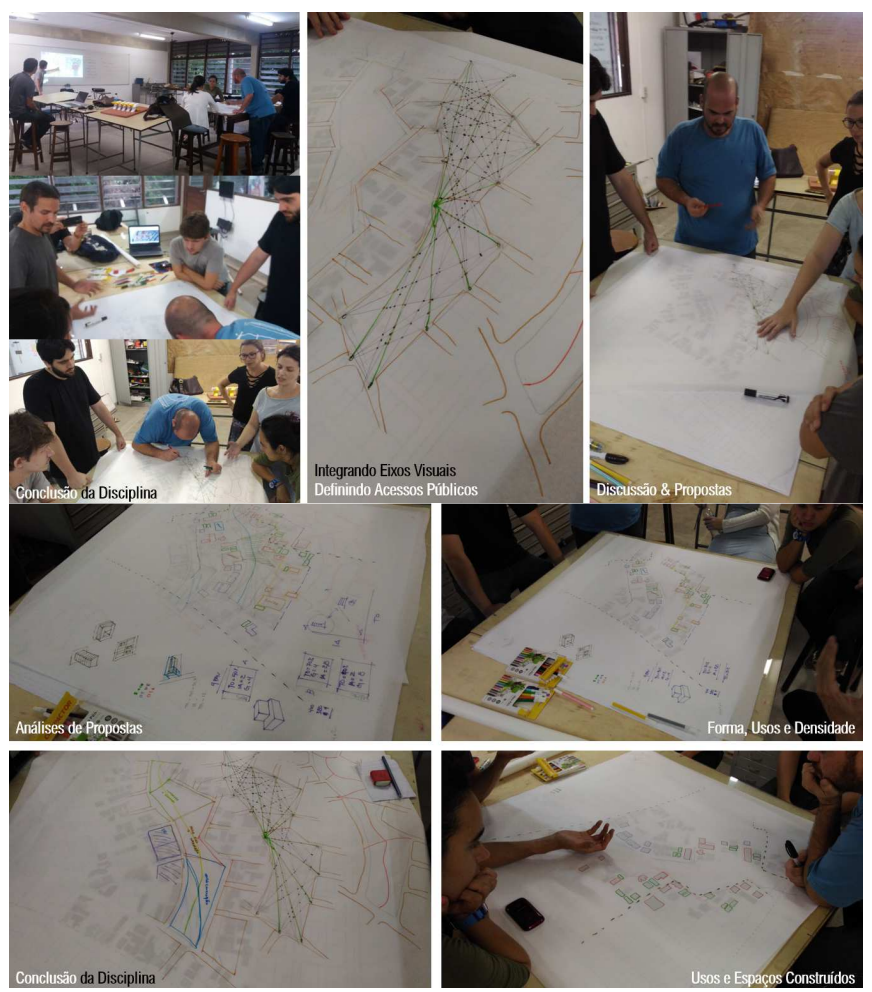

Figura 3 Realização da oficina de desenho urbano no final da disciplina, para uma área pública selecionada do bairro Oceania em João Pessoa-PB. Fonte: Autores (2017).

\section{Discussão}

Dentre alguns resultados notou-se, mais claramente que no momento de escolha dos bairros, os contrastes entre as áreas de estudo escolhidas em João Pessoa, e a existência de problemas distintos para cada caso, exigindo estratégias específicas.

Uma porção do bairro Tambaú pouco mudou quando se analisou os campos visuais com e sem muros: esta área é realmente uma das mais movimentadas dentre as estudadas e apresenta muitos usos comerciais, fachadas ativas e maior densidade (Figura 4). Neste sentido, o bairro Aeroclube foi o contrário, sendo o caso em que campos visuais mais mudaram quando se tiraram os muros e se deixaram apenas as edificações: apresentou densidade baixa em edificações altas e destacadas do espaço público, com poucos usos não residenciais. O bairro Valentina ficou em uma situação intermediária, em que alguns usos comerciais se misturam a edificações de baixo porte e contíguas.

Análises do entorno do bairro Valentina mostram que usos comerciais se concentram em áreas mais acessíveis do sistema, tirando proveito de um maior movimento potencial (Figura 5). Em Tambaú áreas de maior acessibilidade física e visual parecem ser os espaços mais movimentados, correspondendo a áreas com interfaces mais ativas entre espaço público e privado.

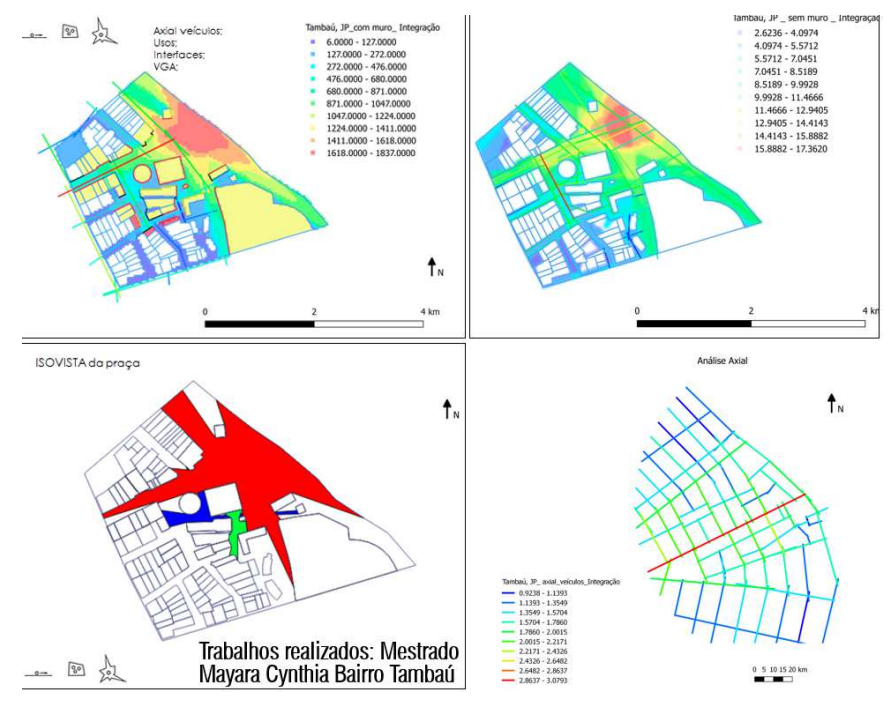

Figura 4 Estudos de campos visuais (escala reduzida) e integração da malha (escala do setor do bairro) de Tambaú, João Pessoa-PB. Fonte: Mayara Cynthia/Adaptado pelos autores (2017).

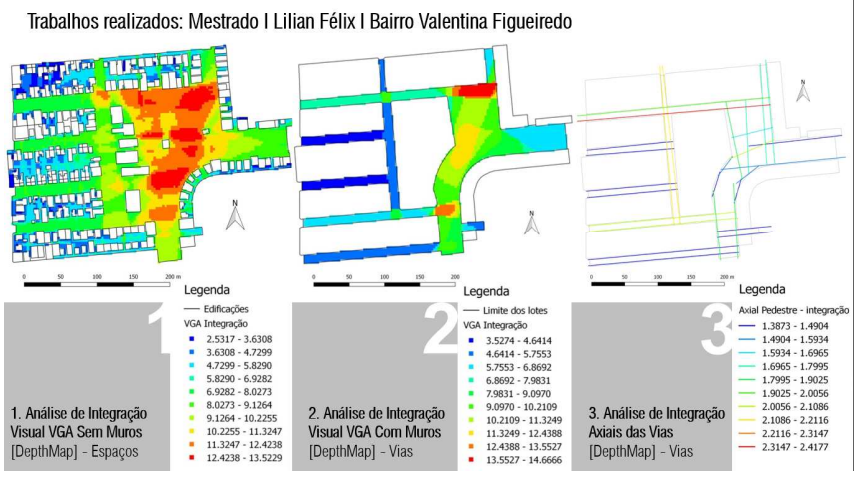

Figura 5 Amostra do bairro Valentina em João Pessoa-PB, simulações de sintaxe espacial. Fonte: Lilian Félix/Adaptado pelos autores (2017).

O estudo de campos visuais e de movimento guiou as intervenções estudadas no bairro Oceania, em que se notou uma concentração de acessibilidade para movimentos de pedestres ao norte da praça e a inexistência de caminhos para 
pedestres para o lado sul, e assim também uma área muito central visualmente, mas até agora impossível de percorrer; sugeriu-se na oficina a abertura de caminhos e pontes no canal, melhorando conexões entre áreas do entorno mais comerciais.

Experiências breves renderam frutos que devem ser desenvolvidos e solidificados para alcançarem validade científica. Todavia, o exercício de analisar alternativas às quadras urbanas atuais, com e sem muros, demonstrou ser um bom exercício de reflexão sobre a análise urbana associada às estratégias de adensamento, ativação das fachadas e implementação de usos mistos, sendo este último que mais eficaz em pontos mais integrados do bairro.

\section{Considerações Finais}

O processo demonstrou a validade de análises de temáticas e escalas diversas inter-relacionadas no campo da pesquisa em arquitetura e urbanismo para melhorar a compreensão das complexidades urbanas, além de ensaiar cenários tendenciais e alternativos. A experiência implementada no PPGAU-UFPB nesta disciplina de mestrado, demonstrou 0 interesse (discente e docente) pela análise configuracional do espaço, focando temáticas variadas de pesquisa em arquitetura e urbanismo, e agregando docentes e alunos com diversas experiências e domínios. A experiência consolida o processo de aprendizado por um lado, e por outro, possibilita propostas mais complexas, em decorrência das distintas visões e expertises do grupo. As experiências descritas devem extrapolar para o cotidiano profissional em escritórios de arquitetura e urbanismo e de planejamento urbano; em outros contextos diversos (Karimi, 2012) se mostrou bem-sucedido.

Os mestrandos analisaram o espaço em relação a campos prováveis de movimento em escalas distintas (local veicular e de pedestres na área pública em foco) e campos visuais em situações distintas (p.ex. com e sem muros), podendo assim se vislumbrar uma outra composição de bairro, mais denso, caminhável, e com espaços de maior vivência humana.

Contudo, cabem ressalvas: 1) Pouco tempo para desenvolvimento das análises e para relacionar com dados do conjunto construído, densidade e usos mistos (módulo 1); 2) O processamento dos dados envolve o conhecimento de outros programas (QGIS e Depthmap), e foi identificada limitações do processamento (p. ex. tempo das análises de integração visual); 3) Softwares complexos de Modelagem Paramétrica foram introduzidos ao grupo (Figura 6), de forma incipiente, pois nenhum aluno tinha conhecimento prévio destes, impossibilitando o avanço da ferramenta. 4) desenvolvimento de processos de programação urbanística baseado em critérios de desempenho urbano.

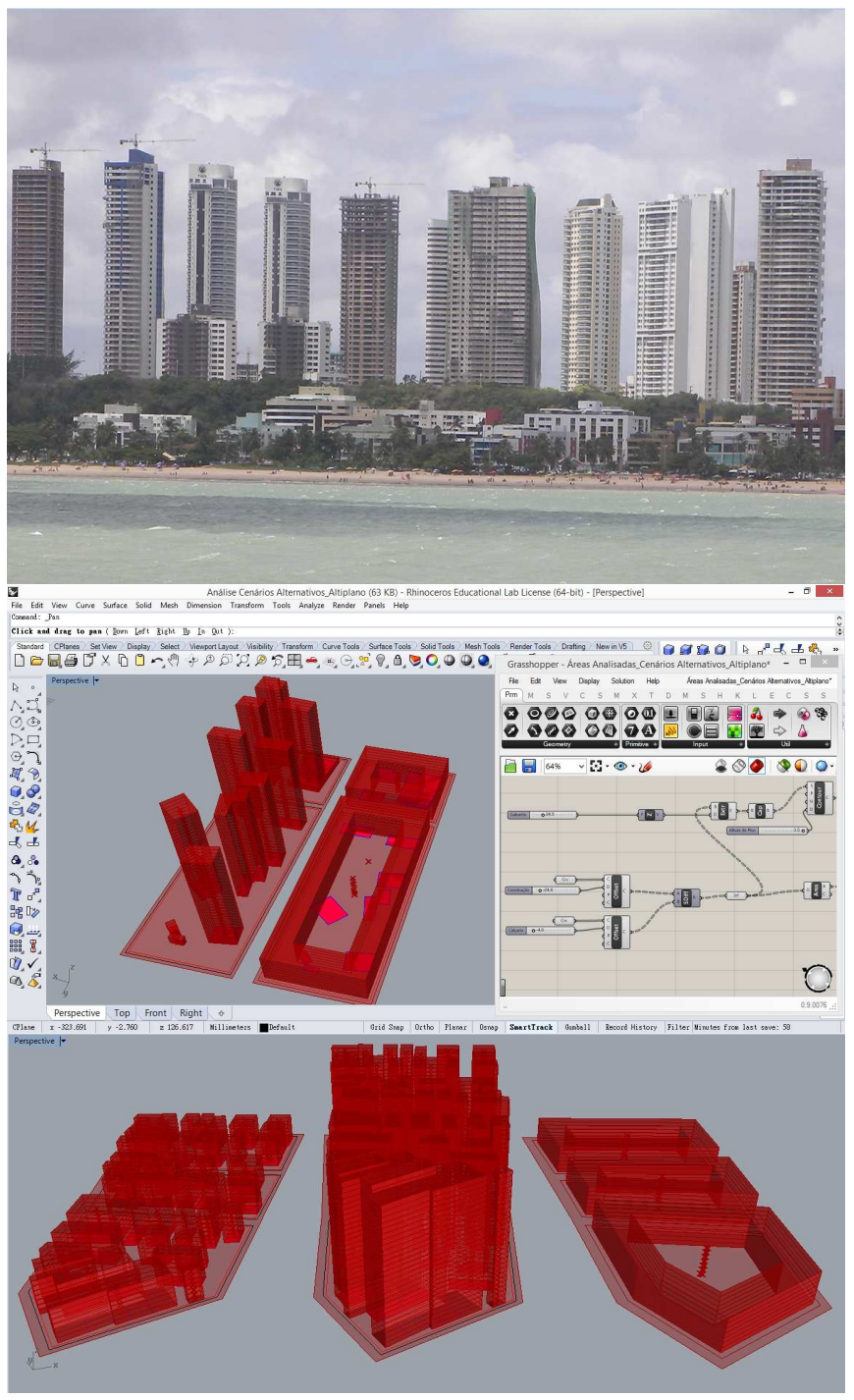

Figura 6 Cenário atual (acima) e simulações em 3D para o bairros de João Pessoa-PB, possibilitando a visualização imediata de cenários alternativos. Fonte: Geovany J. A. Silva (2017).

Deve-se, portanto, 1) Buscar a ampliação e a atualização das bases de dados para análises mais aprofundadas (p.ex. em bases vetoriais georeferenciadas e mapas axiais, de João Pessoa e outras cidades); 2) Prover disciplinas e/ou oficinas de ferramentas específicas, ministrando a sintaxe espacial, ferramentas de análise arquitetônica em meio SIG e/ou Rhinoceros/Grasshopper na graduação e na pós-graduação, possibilitando futuros desenvolvimentos para a pesquisa e projeto em arquitetura e urbanismo. Estas ações estão em curso e continuarão a ser desenvolvidas. 


\section{Referências}

Alexander, Christopher. (1966). A city is not a tree. Design, London: Council of Industrial Design, $n^{\circ}$ 206, 1966.

Alexander, Christopher; Ishikawa, Sara; Silverstein, Murray (2013). Uma linguagem de Padrões / A Pattern Language. Porto Alegre: Bookman, 2013.

Bart, István László. (2010) Urban sprawl and climate change: A statistical exploration of cause and effect, with policy options for the EU. Land use policy, v. 27, n. 2, p. 283-292, 2010.

Berghauser Pont, Meta.; Haupt, Per. (2010). Spacematrix: Space, Density and Urban Form. Rotterdam: NAI Publishers, 2010.

Berry, B. J. L., \& Okulicz-Kozaryn, A.. (2009) Dissatisfaction with city life: A new look at some old questions. Cities, 26, 117-124, 2009.

Berry, B. J. L., \& Okulicz-Kozaryn, A.. (2011) An urban-rural happiness gradient. Urban Geography, 32, 871-883, 2011.

Bertaud, A.; Malpezzi, S. (2003). The Spatial Distribution of Population in 48 World Cities: Implications for Economies in Transition, In Alain Bertaud Web Page.

Camagni, Roberto; Gibelli, Maria Cristina; Rigamonti, Paolo. (2002) Urban mobility and urban form: the social and environmental costs of different patterns of urban expansion. Ecological economics, v. 40, n. 2, p. 199-216, 2002.

Carmona, Matthew; Heath, Tim; Taner, Oc; Tiesdell, Steve. (2007) Public Places - Urban Spaces: The Dimensions of Urban Design. Oxford: Linacre House/Jordan Hill, 2007.

Carmona, Matthew; Tiesdell, Steve. (2003) Urban Design Reader. EUA: Publish by Elsevier Ltda., 2003.

Coelho, Carlos D. (2014). Cadernos de Morfologia Urbana: Os Elementos urbanos (Vol I). / Org.: Carlos D. Coelho. Lisboa, Portugal: Ed. Argumentum, 2014.

Duany, Andres; Plater-Zyberk, Elizabeth; Speck, Jeff. (2001) Suburban Nation: The Rise of Sprawl Suburban and the Decline of Nation the American Dream. Nova York: North Point Press, 2001.

Farr, Douglas. (2013). Urbanismo Sustentável. Porto Alegre: Bookman, 2013.

Frank, Lawrence D. et al. (2006) Many pathways from land use to health: associations between neighborhood walkability and active transportation, body mass index, and air quality. Journal of the American Planning Association, v. 72, n. 1, p. 75-87, 2006.

Frumkin, Howard. (2002) Urban sprawl and public health. Public health reports, v. 117, n. 3, p. 201, 2002.

Gehl, Jan. (2014) Ciudad para la gente. Buenos Aires: Infinito, 2014.

Glaeser, Edward L.; Kahn, Matthew E. (2004) Sprawl and urban growth. Handbook of regional and urban economics, v. 4, p. 24812527, 2004.

Hillier, B. (1996). Space is the machine: a configurational theory of architecture. London, UK: Space Syntax. Retrieved from http://eprints.ucl.ac.uk/3881/
Hillier, B. (2009). Spatial sustainability in cities: Organic patterns and sustainable forms (p. K01:1-K01:20). Presented at the 7th International Space Syntax Symposium, Stockholm: D. Koch, L. Marcus and J. Steen.

Hillier, B., \& Hanson, J. (1984). The social logic of space. Cambridge, England: Cambridge University Press.

Hillier, B., Burdett, R., Peponis, J., \& Penn, A. (1987). Creating Life: Or, Does Architecture Determine Anything? Architecture et Comportement/Architecture and Behaviour, 3(3), 233-250.

Hillier, B.; Vaughan, L. The city as one thing. Progress in Planning, v. 67, n. Elsevier, p. 205-230, 2007.

Jacobs, J. (1992). The death and life of great American cities. New York: Vintage Books.

Karimi, K. (2012). A configurational approach to analytical urban design: 'Space syntax' methodology. URBAN DESIGN International, 17(4), 297-318. https://doi.org/10.1057/udi.2012.19

Karssenberg, Hans et al (2015) A cidade ao nível dos olhos: lições para os plinths. - Porto Alegre : EDIPUCRS, 2015.

Kenworthy, Jeffrey R.; Laube, Felix B. (1999) Patterns of automobile dependence in cities: an international overview of key physical and economic dimensions with some implications for urban policy. Transportation Research Part A: Policy and Practice, v. 33, n. 7, p. 691-723, 1999.

Knight, J., \& Gunatilaka, R. (2010) The rural-urban divide in China: Income but not happiness? Journal of Development Studies, 46, 506-534, 2010.

Li, Norman P. \& Kanazawa, Satoshi (2016) Country roads, take me home. . . to my friends: How intelligence, population density, and friendship affect modern happiness. British Journal of Psychology (2016), 107, 675-697.

Mostafavi, Mohsen. (2014). Urbanismo Ecológico. São Paulo: Gustavo Gili, 2014.

Newman, Peter G.; Kenworthy, Jeffrey R. (1989) Cities and automobile dependence: An international sourcebook. 1989.

Newman, Peter; Kenworthy, Jeffrey. (1999) Sustainability and cities: overcoming automobile dependence. Island press, 1999.

Parham, E. The Segregated Classes: spatial and social relationships in slums. In: Santiago de Chile, PUC. Anais... In: EIGHTH INTERNATIONAL SPACE SYNTAX SYMPOSIUM. Santiago de Chile, PUC: M. Greene, J. Reyes, A. Castro, 2012.

Sennet, R. (2008). The Public Realm. Unpublished Paper for QUANT, http://www.richardsennet.com/.

Serra, M. L. A.; Pinho, P. Tackling the Structure of Very Large Spatial Systems - Space Syntax and the Analysis of Metropolitan Form. The Journal of Space Syntax, v. 4, n. 2, p. 179-196, 26 dez. 2013.

Van de Hoek, Joost W. (2008) The MXI (Mixed-use Index) as Tool for Urban Planning and Analysis. (Paper 03) In Corporations and Cities: Envisioning Corporate Real Estate in the Urban Future. Available 26 May 2008. Delft, Holanda: TU Delft, 2008. Disponível em: www.corporationsandcities.org

Villaça, F. (2001). Espaço intra-urbano no Brasil. São Paulo: Studio Nobel: FAPESP 